\title{
ACTUALIZACIÓN Y CONTROVERSIAS EN EL TRATAMIENTO DE LAS MALFORMACIONES CONGÉNITAS DE LA VÍA AÉREA PULMONAR
}

\author{
UPDATE AND DISAGREEMENTS IN THE TREATMENT OF CONGENITAL PULMONARY \\ AIRWAY MALFORMATIONS \\ Dr. Juan Carlos Pattillo S. ${ }^{1}$, Dra. Josefina Sáez B. ${ }^{1}$, Dr. Fernando Vuletin S. ${ }^{1}$, Interna Isabella Montero J. ${ }^{2}$ \\ 1.- Sección Cirugía Pediátrica, División de Cirugía, Escuela de Medicina, Facultad de Medicina, Pontificia Universidad Católica de Chile. \\ 2.- Interna, Facultad de medicina, Pontificia Universidad Católica de Chile.
}

\section{ABSTRACT}

Congenital pulmonary airway malformations (CPAM) are rare and occur due to a failure in lung embryological development. They are classified according to their pathological characteristics and their anatomical origin. They can occur from the antenatal period to adulthood, can be associated with hydrops fetalis, respiratory distress, recurrent infections, or in an otherwise asymptomatic patient. In this article we carry out a scope review of the literature to answer frequent questions of the clinical teams in charge of patients with CPAM, such as the antenatal approach, the different types of surgeries and the management of asymptomatic patients. Although the indication for surgery is clear in symptomatic patients, little is known about its natural history of this condition, including the possibility of spontaneous resolution and the development of complications or neoplasm. So, the treatment of asymptomatic patients continues to be controversial. Therapeutic decisions must be made by multidisciplinary teams with the informed participation of parents and patients.In our opinion, considering the excellent results of minimally invasive surgery, its low incidence of complications, and practically zero mortality when performed by experienced groups, it seems reasonable to consider elective resection of a MCVAP in asymptomatic patients.

Key words: Congenital abnormalities, cystic adenomatoid malformation of lung, lobectomy, VATS.

\section{RESUMEN}

Las malformaciones congénitas de la vía aérea pulmonar (MCVAP) son infrecuentes y ocurren debido a una falla en el desarrollo embriológico pulmonar. Se clasifican de acuerdo con sus características patológicas y a su origen anatómico. Se pueden presentar desde el periodo antenatal hasta la adultez, asociarse a cuadros de hidrops fetal, distrés respiratorio, infecciones recurrentes, 0 como un hallazgo en pacientes asintomáticos. En este artículo realizamos una revisión bibliográfica exploratoria para responder dudas frecuentes de los equipos clínicos a cargo de pacientes con MCVAP, como el enfrentamiento antenatal, los distintos tipos de cirugía y su abordaje, y el manejo de pacientes asintomáticos. Si bien la indicación de cirugía es clara en pacientes sintomáticos, poco se conoce acerca de su historia natural, incluyendo la posibilidad de resolverse de forma espontánea, de complicarse o de evolucionar hacia el desarrollo de una neoplasia, por lo que el tratamiento de pacientes asintomáticos continúa siendo controversial. Las decisiones terapéuticas deben ser tomadas por equipos multidisciplinarios con la participación informada de los padres y de los pacientes. En nuestra opinión, considerando los excelentes resultados de la cirugía mínimamente invasiva, su baja incidencia de complicaciones y prácticamente nula mortalidad, al ser realizada por grupos con experiencia, nos parece razonable plantear la resección electiva de una MCVAP en un paciente asintomático. Palabras claves: Malformaciones congénitas, malformación quística pulmonar, lobectomía, toracoscopía.

\section{INTRODUCCIÓN}

Las lesiones quísticas pulmonares representan un espectro de condiciones que pueden ser congénitas o adquiridas, pueden presentarse desde el período antenatal, y ocurrir como un hallazgo asintomático o con alguna complicación clínica.

Dentro de las lesiones quísticas congénitas, que ocurren por una falla en algún momento del desarrollo embriológico pulmonar, podemos encontrar las malformaciones congénitas de la vía aérea pulmonar (MCVAP), anteriormente

\section{Correspondencia:}

Dr. Juan Carlos Pattillo Silva

Sección Cirugía Pediátrica, División de Cirugía, Escuela de Medicina, Facultad de Medicina, Pontificia Universidad Católica de Chile.

Diagonal Paraguay 362, Santiago. Chile

jpattill@uc.cl descritas como "malformaciones adenomatoideas quísticas", los quistes broncogénicos, quistes de duplicación intestinal y Ios quistes periféricos asociados a la trisomía 21. Los quistes adquiridos se observan después de la resolución de un proceso inflamatorio y de necrosis pulmonar, que luego epiteliza, dejando una cavidad residual.

En este artículo nos enfocamos en las MCVAP, con una breve actualización de su clasificación y una discusión acerca de las alternativas de tratamiento en distintos escenarios clínicos, lo que sin duda constituye un punto de controversia y un desafío para los equipos clínicos a cargo de este grupo de pacientes.

\section{EMBRIOLOGÍA Y CLASIFICACIÓN}

Si bien los primeros casos descritos de malformaciones quísticas pulmonares datan del siglo XIX, no fue hasta 1949 
en que Chin y Tang proponen el nombre de "malformación adenomatoidea congénita" para describir una malformación pulmonar de aspecto adenomatoide (1). Posteriormente, se incluyeron otras lesiones pulmonares congénitas similares a esta, pero con epitelio bronquiolar y un patrón hamartomatoso, siendo entonces conocidas como "malformación adenomatoidea quística". En 1977 Stocker (2) las clasifica en tres grupos, basándose en las características patológicas de estas lesiones. Las lesiones tipo I, que corresponden a lesiones quísticas grandes cubiertas de epitelio y que provocan dificultad respiratoria al Ilenarse de aire al momento del nacimiento (Figura 1), las tipo II, compuestas de estructuras bronquiolares, asociadas a otras malformaciones, y las tipo III, que corresponden a las malformaciones adenomatoideas descritas originalmente por Chin y Tan. Las observaciones iniciales de Stocker fueron posteriormente complementadas (3), agregando otros dos grupos: malformaciones no quísticas de la tráquea y bronquios, y lesiones quísticas cubiertas por neumocitos tipo 1 y 2. Como la numeración de las malformaciones representa la región anatómica en la que ocurren, y con el objetivo de mantener la numeración original de la clasificación, se propuso cambiar la nomenclatura a números arábigos (ya que los números romanos no tienen ceros) e iniciarla desde cero con las malformaciones más proximales (Tabla 1).

Figura 1. Radiografía de tórax AP y $L$ (ay b) en que se observa una imagen radiolúcida en el lóbulo superior izquierdo (flecha negra). En la tomografía computada de tórax (c) se confirma la presencia de una lesión quística unilocular, de paredes finas y bien definidas, compatible con una MCVAP tipo 1.

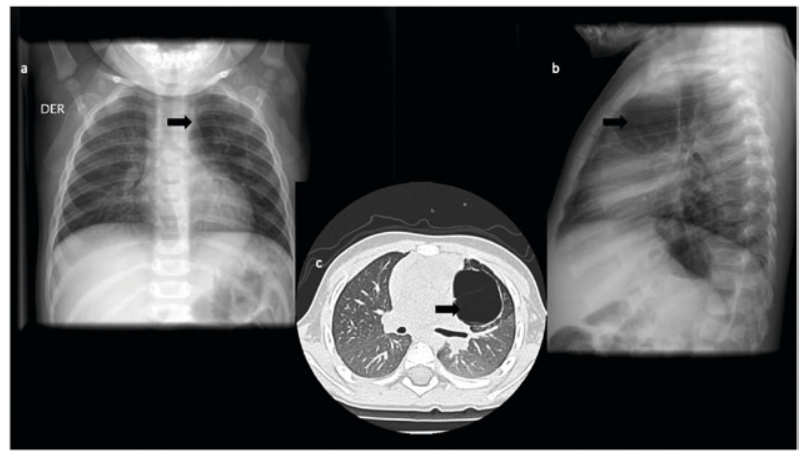

\section{ESTRATEGIAS DE TRATAMIENTO}

La presentación clínica de las MCVAP puede ser variable. Grandes lesiones que provocan efecto de masa pueden ocasionar desviación de las estructuras mediastínicas, polihidramnios e hidrops fetal (4). Otras pueden manifestarse con síndrome de dificultad respiratoria en el período neonatal o como infecciones recurrentes en la infancia (4).

La amplia disponibilidad y el uso rutinario, además del desarrollo cualitativo de la ecografía obstétrica, han permitido un aumento en la detección prenatal de las MCVAP (5). Incluso algunos autores refieren que la incidencia estimada
Tabla 1. Clasificación de las malformaciones pulmonares congénitas de la vía aérea pulmonar

\begin{tabular}{|c|c|c|c|c|c|}
\hline & Incidencia & Ubicación & $\begin{array}{l}\text { Tamaño de } \\
\text { los quistes }\end{array}$ & Histología & Notas \\
\hline 0 & infrecuente & $\begin{array}{l}\text { Tráqueo- } \\
\text { bronquial }\end{array}$ & No quistica & $\begin{array}{l}\text { Falla completa en el } \\
\text { desarrollo después de } \\
\text { la etapa } \\
\text { pseudoglandular }\end{array}$ & $\begin{array}{l}\text { Displasia acinar } \\
\text { congénita (letal) }\end{array}$ \\
\hline 1 & $\begin{array}{l}\text { Frecuente } \\
50 \%\end{array}$ & $\begin{array}{l}\text { Bronquial- } \\
\text { Bronquiolar }\end{array}$ & $\begin{array}{l}>2 \mathrm{~cm}, \text { puede } \\
\text { ser múltiple }\end{array}$ & $\begin{array}{l}\text { Estructura alveolar } \\
\text { dilatada, con escaso o } \\
\text { mínimo componente } \\
\text { adenomatoide. } \\
\text { Epitelio columnar } \\
\text { pseudoestratificado } \\
\text { ciliado, con } \\
\text { interposición de } \\
\text { células mucosas. }\end{array}$ & \\
\hline 2 & $\begin{array}{l}\text { Frecuente } \\
0 \%-45 \%\end{array}$ & Bronquiolar & $\begin{array}{l}\text { Múltiples } \\
\text { quistes } \\
\text { pequeños, } \\
\text { "esponja" }\end{array}$ & $\begin{array}{l}\text { Estructuras de } \\
\text { aspecto bronquial } \\
\text { dilatadas con } \\
\text { interposición de } \\
\text { parénquima alveolar. } \\
\text { Presencia ocasional } \\
\text { de músculo estriado. }\end{array}$ & $\begin{array}{l}\text { Asociado a } \\
\text { malformaciones } \\
\text { genitourinarias } \\
\text { y } \\
\text { gastrointestinal } \\
\text { es. }\end{array}$ \\
\hline 3 & $\begin{array}{l}\text { Infrecuente } \\
5 \%\end{array}$ & $\begin{array}{l}\text { Bronquiolar- } \\
\text { Alveolo ductal }\end{array}$ & $\begin{array}{l}\text { Sólido } \\
\text { (quistes } \\
\text { menores a } \\
3 \mathrm{~mm} \text { ) }\end{array}$ & $\begin{array}{l}\text { Estructuras } \\
\text { bronquiolares } \\
\text { separadas por } \\
\text { espacios aéreos } \\
\text { pequeños cubiertos } \\
\text { por epitelio cuboidal, } \\
\text { similar al pulmón fetal } \\
\text { tardio. }\end{array}$ & \\
\hline 4 & $\begin{array}{l}\text { Muy } \\
\text { infrecuente }\end{array}$ & Acinar distal & $\begin{array}{l}\text { Quistes } \\
\text { grandes }\end{array}$ & $\begin{array}{l}\text { Quistes periféricos } \\
\text { con epitelio alveolar o } \\
\text { bronquial sobre tejido } \\
\text { mesenquimatoso laxo }\end{array}$ & $\begin{array}{l}\text { Se relaciona } \\
\text { con blastoma } \\
\text { pleuropulmonar } \\
\text { involucionado } \\
\text { tipo } 1\end{array}$ \\
\hline
\end{tabular}

previamente en 1/30.000 recién nacidos vivos, podría elevarse hasta 1/7.000 (4). Lo anterior ha motivado preguntas respecto de la verdadera historia natural de estas lesiones, pues una proporción importante sería asintomática, mientras que otras podrían resolverse después del nacimiento. Además, ha existido algún grado de controversia respecto de la relación de estas malformaciones y el desarrollo de cáncer en el largo plazo (6). Por lo tanto, existen interrogantes en cuanto a las mejores estrategias de tratamiento en los distintos grupos etarios y de acuerdo con sus formas de presentación, las que discutiremos a continuación.

\section{Estrategias de tratamiento antenatal}

Una MCVAP suele identificarse en los estudios ecográficos antenatales entre las 18 y las 21 semanas de gestación (5). Las MCVAP pueden comprometer cualquier lóbulo, habitualmente son unilaterales y su irrigación depende de la circulación pulmonar, aunque es frecuente encontrar lesiones mixtas, que incluyen un componente de secuestro 
con irrigación sistémica (7). La mayoría de estas lesiones son asintomáticas y sólo requieren de seguimiento, sin embargo, aquellas lesiones de gran tamaño pueden provocar desviación mediastínica y aplanamiento del diafragma ipsilateral (7). Algunos pacientes presentan polihidramnios, explicado por compresión esofágica o sobreproducción de líquido en la lesión, e incluso hidrops fetal no inmune, secundario a la compresión cardíaca $\mathrm{y} / 0$ de la vena cava inferior lo que se reporta en alrededor del $5 \%$ de los pacientes con diagnóstico antenatal de MCVAP referidos a centros terciarios (5).

La resonancia magnética fetal puede ser complementaria, contribuyendo al diagnóstico diferencial de lesiones intratorácicas y precisando los hallazgos ecográficos $(8,9)$. (Figura 2).

Figura 2. Imágenes de una Resonancia fetal en corte coronal (a) y axial a nivel del tórax fetal (b) en que se observa hiperexpansión del pulmón izquierdo (flecha negra) secundario a una lesión hiperintensa constituida por múltiples imágenes quísticas menores a $5 \mathrm{~mm}$ (asteriscos) compatible con una MCVAP tipo 2, Hay además compresión del pulmón derecho (flechas blancas).

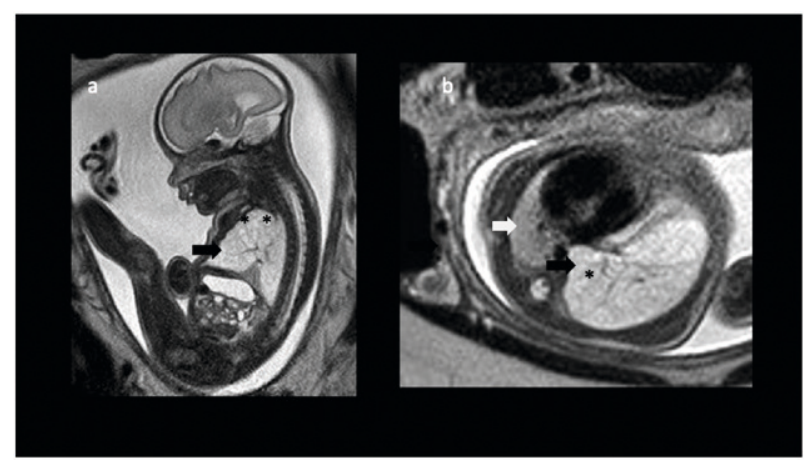

La historia natural in-útero varía desde lesiones con crecimiento rápido que pueden derivar en hidrops y en un mal pronóstico fetal, a aquellas con comportamiento regresivo parcial o total (5). El índice CVR (Cystic adenomatoid malformation Volume Ratio), que corresponde al cálculo del volumen de la lesión (de acuerdo a la fórmula para una elipse), normalizado por la edad gestacional al dividir por la circunferencia de la cabeza, se ha planteado como predictor del outcome de estos pacientes (10). Un CVR menor a 1.6 se relaciona con sobrevidas mayores al $90 \%$ y un riesgo menor al 3\% de desarrollar hidrops (10).

El seguimiento ecográfico riguroso permitirá definir la conducta terapéutica y aconsejar a los padres, de acuerdo al comportamiento de la masa, la condición del feto y la edad gestacional (11). Una lesión que mantiene o disminuye su tamaño, que presenta un CVR de bajo riesgo, podría solo observarse.

La aparición de hidrops determina la necesidad imperativa de intervención, pues el manejo expectante resulta en la muerte del feto (11). No se ha definido un límite exacto de edad gestacional para plantear interrupción del embarazo y resección posnatal, versus intervención prenatal. Dependerá de la experiencia en los distintos centros, pero se sugiere en aquellos fetos sin otras anomalías asociadas, que desarrollan hidrops después de las 30-32 semanas, interrumpir el embarazo previa administración de betametasona, pues aumentaría la sobrevida (11).

La decisión de realizar una intervención in-útero debe considerar el comportamiento de la MCVAP, la posición del feto y la placenta, el riesgo de parto prematuro por la presencia de polihidramnios, el desarrollo de hidrops fetal y la hipoplasia pulmonar. Además de los recursos técnicos disponibles.

Entre las alternativas terapéuticas se ha descrito la toracocentesis o punción y drenaje de un quiste dominante de manera repetida, pero como intervención única tendrían poca utilidad pues la acumulación de líquido suele reproducirse (11). La instalación de un shunt toraco amniótico ha mostrado resultados favorables en lesiones macroquísticas, aunque el dispositivo puede migrar, ocluirse o disfuncionar $(12,13)$. La escleroterapia de la lesión dirigida por ecografía 0 el uso de radiofrecuencia o láser percutáneo para suprimir el flujo sanguíneo a la malformación, se han reportado en casos aislados y con resultados variables $(11,14,15)$. La broncoscopia fetal y la cirugía fetal abierta para realizar una Iobectomía se realizan solo en algunos centros, como salvataje y con resultados positivos en escenarios extremos (11, 16). Por último, los corticoides administrados a la madre en el segundo trimestre del embarazo, han demostrado utilidad en provocar regresión de la lesión y resolución del hidrops en malformaciones predominantemente microquísticas $(17,18)$.

Aquellos pacientes con diagnóstico antenatal requieren seguimiento del embarazo, además de considerar la planificación del parto y el cuidado del recién nacido. Deberían ser derivadas a centros terciarios, con acceso a unidad de cuidados intensivos neonatales, cirugía pediátrica e incluso fetal si estuviera disponible.

\section{Estrategias de tratamiento neonatal de las MCVAP}

El paciente con diagnóstico antenatal de MCVAP que requirió interrupción del embarazo por complicación fetal, debe ser estabilizado e intervenido en el período neonatal. Un grupo seleccionado, en el que se identifica que la malformación pone en riesgo la oxigenación, podría ser candidato al procedimiento EXIT (ex-utero intrapartum therapy) en el momento del parto. En él, el feto es parcialmente exteriorizado y se establece control de la vía aérea y acceso endovenoso, para luego proceder a la toracotomía y lobectomía, mientras aún existe soporte materno-placentario (11). Esta aproximación permitiría evitar el escenario de un recién nacido inestable, persistentemente hipóxico, que requiere toracotomía de emergencia y resección de la masa. Quien además está expuesto a presentar desde descompensación hemodinámica, a expansión de la lesión por atrapamiento aéreo 0 neumotórax espontáneo.

Aquellos casos en que el seguimiento ecográfico prenatal consideró estabilidad o regresión de la lesión y llegaron a término, pueden o no ser sintomáticos en el período neonatal. Se discute si las lesiones con comportamiento regresivo realmente desaparecen o solo disminuyen su tamaño 
relativo con el crecimiento del pulmón en desarrollo (4).

Existe consenso en indicar la cirugía en aquellos pacientes con distrés respiratorio, pero no así en los que no tienen repercusión clínica. Una tomografía computada (TAC) de tórax de alta resolución es útil para una caracterización anatómica detallada y se sugiere en el período postnatal (9), en aquellos casos en que se plantea un abordaje quirúrgico 0 bien para objetivar la presencia de una lesión cuando persiste la duda diagnóstica. Sin embargo, puede diferirse para los primeros meses de vida cuando el paciente es asintomático.

\section{¿Toracotomía o Cirugía Mínimamente Invasiva? ¿Lobec- tomía pulmonar o resección segmentaria?}

La lobectomía pulmonar es una cirugía que puede ser realizada por toracotomía o abordaje mínimamente invasivo. La intervención abierta se suele preferir en pacientes que se operan durante las primeras semanas de vida y también en aquellos con distrés respiratorio que se considera no tolerarán ventilación mono bronquial o insuflación en el hemitórax comprometido (19). La lobectomía por toracoscopia video asistida es una alternativa segura en manos de equipos con experiencia y aunque la evidencia es principalmente retrospectiva, se considera que suele asociarse a menor tiempo operatorio, estadía hospitalaria, requerimiento de analgesia, menos secuelas estéticas y una tasa de complicaciones equivalente 0 incluso menor que la cirugía abierta (20). Con el avance de la técnica quirúrgica, la mejoría y miniaturización del instrumental disponible y nuevos dispositivos de hemostasia, hoy en día es posible realizar estas cirugías en recién nacidos de forma segura.

En general el tratamiento con una lobectomía anatómica reglada es preferido sobre las resecciones segmentarias, pues ofrece la seguridad de una resección completa, disminuyendo la posibilidad de recidiva. Sin embargo, parece razonable plantear una resección segmentaria en lesiones pequeñas y bien definidas, o bien en lesiones quísticas, sobre todo con quistes dominantes, en que es posible asegurar la resección de la lesión completa (Figura 1). La ventaja de las resecciones parciales por sobre la lobectomía es teórica, y tiene que ver con la menor cantidad de pulmón funcional resecado en una condición benigna (21).

\section{Tratamiento de las MCVAP en pacientes asintomáticos}

El diagnóstico postnatal puede ocurrir en distintos escenarios y a diferentes edades. La presentación puede ser como sintomatología respiratoria de gravedad variable, infecciones pulmonares recurrentes o incluso como hallazgo incidental. Cualquiera sea el caso, precisar el tipo de lesión y sus características requerirá la aproximación con una imagen. Muchas veces será de manera inicial una radiografía de tórax, pero la definición de la anatomía más precisa requerirá un TAC de tórax de alta resolución (9).

La aparición de manifestaciones clínicas justifica plenamente la indicación quirúrgica. Sin embargo, proponer el tratamiento en aquellos pacientes asintomáticos sigue siendo controversial, lo que discutiremos a continuación (22).

\section{¿Es mayor el riesgo de la cirugía o de la observación?}

El mayor argumento para no operar a un paciente asintomático es el potencial riesgo quirúrgico al que es sometido el paciente (Tabla 2). Sin embargo, las tasas de morbilidad reportadas son más bien bajas, sin mortalidad asociada $(23,24)$, y la lobectomía es bien tolerada, sin asociarse a limitaciones funcionales en el largo plazo $(25,26)$. También debe considerarse que la observación activa no ha demostrado peores resultados que el manejo quirúrgico (23), aunque se asocia a mayor exposición a radiación ionizante por el uso de tomografías computadas seriadas de monitorización (23, $24,27)$. A pesar de que el impacto de estos exámenes no ha sido investigado, se plantean como una preocupación en la población pediátrica.

Tabla 2. Resumen de evidencia respecto a estrategias de tratamiento

\begin{tabular}{|c|c|c|}
\hline Discusión & $\begin{array}{c}\text { Favorece la resección } \\
\text { quirúrgica }\end{array}$ & $\begin{array}{l}\text { Favorece la } \\
\text { observación }\end{array}$ \\
\hline $\begin{array}{l}\text { Riesgo de } \\
\text { infección }\end{array}$ & $\begin{array}{l}\text { El índice de complicaciones } \\
\text { en pacientes asintomáticos } \\
\text { es de } 3.2 \% \text { antes de los } 7 \\
\text { meses de vida. (32) }\end{array}$ & $\begin{array}{l}<5 \% \text { de los casos } \\
\text { desarrollarán síntomas } \\
\text { antes de los } 5 \text { años de edad. } \\
\text { (35) }\end{array}$ \\
\hline $\begin{array}{l}\text { Riesgo de } \\
\text { progresión } \\
\text { neoplásica }\end{array}$ & $\begin{array}{l}\text { El riesgo de neoplasia se } \\
\text { estima entre un } 1 \text { y } 3 \% \text {. Sólo } \\
\text { la resección permite análisis } \\
\text { de anatomía patológica para } \\
\text { alcanzar un diagnóstico } \\
\text { definitivo y descartar } \\
\text { neoplasia en casos de duda } \\
\text { diagnóstica. (6) }\end{array}$ & $\begin{array}{l}\text { La progresión maligna de un } \\
\text { MCVAP antenatal se limita a } \\
\text { casos anecdóticos, a } \\
\text { diferencia de lesiones que se } \\
\text { presentan de manera } \\
\text { postnatal. (35) }\end{array}$ \\
\hline $\begin{array}{l}\text { Función } \\
\text { pulmonar }\end{array}$ & $\begin{array}{l}\text { La lobectomía precoz tendría } \\
\text { mejores resultados en cuanto } \\
\text { a desarrollo pulmonar } \\
\text { compensatorio, siendo } \\
\text { similar a controles sanos } \\
\text { (36). }\end{array}$ & $\begin{array}{l}\text { No habria diferencias en } \\
\text { cuanto a función pulmonar } \\
\text { de pacientes intervenidos de } \\
\text { manera precoz o tardia (28). }\end{array}$ \\
\hline $\begin{array}{l}\text { Riesgo } \\
\text { quirúrgico }\end{array}$ & $\begin{array}{l}\text { La lobectomía no se asocia a } \\
\text { mortalidad, y tiene baja tasa } \\
\text { de morbilidad, es bien } \\
\text { tolerada, sin repercusiones } \\
\text { en función pulmonar. (28) }\end{array}$ & $\begin{array}{l}\text { El abordaje conservador en } \\
\text { pacientes asintomáticos } \\
\text { confiere menor riesgo que los } \\
\text { asociados a una cirugía. (35) }\end{array}$ \\
\hline $\begin{array}{l}\text { Progresión } \\
\text { de } \\
\text { enfermedad }\end{array}$ & $\begin{array}{l}\text { Las complicaciones } \\
\text { quirúrgicas son más } \\
\text { frecuentes en cirugías } \\
\text { realizadas en pacientes } \\
\text { sintomáticos que en } \\
\text { asintomáticos. (28) }\end{array}$ & $\begin{array}{l}\text { El riesgo de que una lesión } \\
\text { se vuelva sintomática es } \\
\text { entre } 3 \text { y } 10 \%(29,35) \\
\text { Existen series con } \\
\text { seguimiento entre } 3 \text { meses y } \\
9 \text { años en que un porcentaje } \\
\text { significativo }(35,7 \%) \text { de } \\
\text { pacientes no presentan } \\
\text { síntomas }(28) \text {. }\end{array}$ \\
\hline
\end{tabular}

Una revisión de la Sociedad Canadiense de Cirujanos Pediátricos identificó nueve estudios no randomizados, solo uno de ellos de diseño prospectivo, con datos agrupables para comparación (28). Se consideraron 168 pacientes asintomáticos al nacimiento, de los cuales $41.7 \%$ fueron sometidos a cirugía electiva con 7 casos (10\%) reportados de complicaciones postoperatorias (28). Del 58.3\% restante, 63 pacientes desarrollaron síntomas entre el mes y los siete años de vida, requiriendo resección quirúrgica y con 20 casos reportados de complicaciones postoperatorias (31.8\%) (28). En el grupo de manejo conservador, los pacientes fueron 
seguidos entre 3 meses y 9 años sin complicaciones (28). El metanálisis reveló que las complicaciones fueron significativamente mayores para el grupo con cirugía luego del desarrollo de síntomas comparado con el grupo asintomático intervenido $(\mathrm{OR} 4.59,95 \% \mathrm{Cl}=1.40$ a $15.11, \mathrm{P}=0.01$; baja heterogeneidad $(I 2=0 \%)$, aunque sin diferencias en la estadía hospitalaria luego de la cirugía (28).

\section{¿Desaparecen las MCVAP en forma espontánea?}

El aumento del diagnóstico antenatal ha permitido seguir de manera prospectiva cohortes de pacientes y entregar ideas respecto a la historia natural de las lesiones, aportando información para poder tomar decisiones y realizar consejería a los padres de pacientes portadores de MCVAP. Una de las experiencias que reporta la evolución de 119 pacientes, informa que $43 \%$ de ellos fueron operados; $6.7 \%$ de emergencia en el período neonatal, $5.1 \%$ de manera electiva por signos de alarma en estudios imagenológicos, 17\% por sugerencia del médico tratante, $0.8 \%$ por infección respiratoria severa y en $4.2 \%$ la indicación no fue clara (29). El 57\% de los pacientes manejados de manera expectante fueron seguidos por una mediana de 9.9 años; $5.9 \%$ tuvieron resolución en las imágenes, $10 \%$ fueron dados de alta, $12.5 \%$ se perdieron del seguimiento y un $76 \%$ continuaba en observación al momento de la publicación (29). Si bien algunos grupos quirúrgicos han sido muy agresivos en la resección precoz de estas lesiones, incluso en el período neonatal (30) parece ser que el seguimiento con imágenes y la resección diferida alrededor de los 3 a 6 meses es una estrategia razonable para evitar resecciones pulmonares innecesarias, minimizar el riesgo perioperatorio y permitir un desarrollo pulmonar compensatorio adecuado (31).

\section{¿Existe riesgo de desarrollar cáncer en una MCVAP?}

Uno de los argumentos para proponer la cirugía es el riesgo de malignidad. Las lesiones quísticas pulmonares se han asociado a dos tipos de neoplasias; el blastoma pleuropulmonar tipo I y el carcinoma bronquioalveolar $(32,33)$. Ambos tumores son muy raros. Algunas características imagenológicas permitirían sospecharlos (estudio anatómico del segundo trimestre normal, crecimiento rápido, lesiones multilobares o bilaterales, neumotórax, historia familiar, lesiones periféricas sin etiología clara, dolor torácico, hemoptisis), así como la presencia de otras neoplasias o alteraciones genéticas. Sin embargo, el diagnóstico definitivo sólo lo hará la anatomía patológica $(32,33)$, lo que podría ser un buen argumento para plantear la resección de una MCVAP. En nuestra experiencia tenemos tres pacientes con MCVAP en los que se diagnosticó finalmente un blastoma pleuropulmonar, un caso comunicado recientemente se presentó como una malformación torácica (34). Se estima que el riesgo de desarrollar cáncer en una MCVAP fluctúa entre un 1\% y un 3\% de estas lesiones (6), Io que para algunos autores justifica la resección quirúrgica.

\section{¿Cuál es la posibilidad de una MCVAP de hacerse sinto- mática?}

El riesgo de que una lesión asintomática desarrolle sintomatología se estima entre un 3 a 10\% $(29,35)$. Los seguimientos prospectivos han evidenciado que el riesgo disminuiría luego del segundo año de vida (29). Sin embargo, algunos trabajos reportan mayor tasa de complicaciones para la cirugía en pacientes sintomáticos y aunque esto puede estar influenciado por aquellos que se intervienen en condiciones más graves en el período neonatal, una lobectomía en un pulmón expuesto a infecciones recurrentes será probablemente en el contexto de mayor inflamación o secuelas de ella (32) (Figura 3). El manejo expectante de las lesiones asintomáticas es sin duda una alternativa a considerar; requiere adherencia del paciente y su familia, además de la repetición de exámenes imagenológicos por un período que aún no ha sido definido y que exponen a radiación ionizante $(29,36)$.

Figura 3. Imágenes de una tomografía computada de tórax en corte axial (a) y reconstrucción coronal (b) sin contraste en que se observan imágenes quísticas de paredes gruesas (flecha negra). En la de mayor tamaño presenta un pequeño nivel en su interior (asterisco), compatible con una MCVAP con signos de sobreinfección.

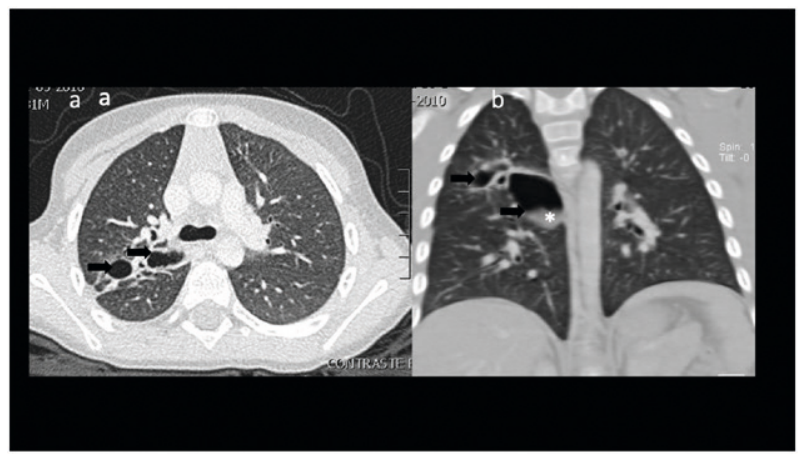

\section{CONCLUSIONES}

Las MCVAP corresponden a un grupo de lesiones que ocurren por fallas en el desarrollo embriológico del pulmón y la vía aérea, cuya clasificación ha evolucionado en la medida que se ha avanzado en la comprensión de su origen embriológico y anatómico. Su enfrentamiento requiere del aporte de distintas especialidades y debe ser realizado por equipos multidisciplinarios.

Basados en los elementos planteados y en la evidencia disponible, en nuestro grupo recomendamos la resección electiva de las MCVAP en pacientes asintomáticos. En Ios pacientes con diagnóstico antenatal realizamos un TAC dentro del primer mes de vida y en el preoperatorio inmediato. Planificamos la cirugía electiva, preferentemente por videotoracoscopía, alrededor de los 6 meses (entre los 3 meses y el año de vida). La resección de una MCVAP permitirá identificar o prevenir el desarrollo de un cáncer, junto con reducir la exposición a radiación ionizante asociada al seguimiento en quienes se decide manejo expectante. Una resección pulmonar en un paciente asintomático es una cirugía segura, que evita el riesgo de aparición de complicaciones 
infecciosas. Además, es una cirugía menos mórbida y técnicamente más sencilla que la realizada en pacientes que han tenido infecciones previas, sobre todo si se realiza por vía mínimamente invasiva.

\section{Agradecimientos}

Los autores agradecen la invaluable colaboración de la Dra. Florencia de Barbieri Magnone, Profesora Asistente del Departamento de Radiología, División de Imágenes, Laboratorios y Patologías, Escuela de Medicina, Pontificia Universidad Católica de Chile, quien ha contribuido con las imágenes y su descripción, resentadas en este artículo.

\section{Los autores declaran no tener conflictos de interés.}

\section{REFERENCIAS BIBLIOGRAFICAS}

1. Ch'in KY, Tang MY. Congenital adenomatoid malformation of one lobe of a lung with general anasarca. Arch Pathol (Chic). 1949, 48(3):221-9.

PMID: 18137795.

2. Stocker JT, Madewell JE, Drake RM. Congenital cystic adenomatoid malformation of the lung. Classification and morphologic spectrum. Hum Pathol. 1977;8(2):15571

doi: 10.1016/s0046-8177(77)80078-6. PMID: 856714.

3. Stocker JT. Cystic lung disease in infants and children. Fetal Pediatr Pathol. 2009;28(4):155-84. doi: 10.1080/15513810902984095. PMID: 19842869.

4. Lau CT, Kan A, Shek N, Tam P, Wong KK. Is congenital pulmonary airway malformation really a rare disease? Result of a prospective registry with universal antenatal screening program. Pediatr Surg Int. 2017;33(1):105108.

doi: 10.1007/s00383-016-3991-1. Epub 2016 Oct 21. PMID: 27770196.

5. Gajewska-Knapik K, Impey L. Congenital lung lesions: Prenatal diagnosis and intervention. Semin Pediatr Surg. $2015 ; 24(4): 156-9$.

doi: $\quad$ 10.1053/j.sempedsurg.2015.01.012. PMID: 26051047

6. Chuang, S., Sugo, E., \& Jaffe, A. A review of postnatal management of congenital pulmonary airway malformations. Fetal and Maternal Medicine Review, 2009 20(3), 179-204. doi:10.1017/S0965539509990052

7. Annunziata F, Bush A, Borgia F, Raimondi F, Montella S, Poeta $M$, et al. Congenital Lung Malformations: Unresolved Issues and Unanswered Questions. Front Pediatr. $201913 ; 7: 239$

doi: 10.3389/fped.2019.00239. PMID: 31249823; PMCID: PMC6584787.

8. Epelman M, Kreiger PA, Servaes S, Victoria T, Hellinger JC. Current imaging of prenatally diagnosed congenital lung lesions. Semin Ultrasound CT MR. 2010; 31(2):14157. doi: 10.1053/j.sult.2010.01.002. PMID: 20304322.
9. Chowdhury MM, Chakraborty S. Imaging of congenital lung malformations. Semin Pediatr Surg. 2015; 24(4):168-75.

doi: $\quad$ 10.1053/j.sempedsurg.2015.02.001. PMID: 26051049.

10. Crombleholme TM, Coleman B, Hedrick H, Liechty $K$, Howell L, Flake AW, et al. Cystic adenomatoid malformation volume ratio predicts outcome in prenatally diagnosed cystic adenomatoid malformation of the lung. J Pediatr Surg. 2002; 37(3):331-8. doi: 10.1053/ jpsu.2002.30832. PMID: 11877643.

11. Mann S, Wilson RD, Bebbington MW, Adzick NS, Johnson MP. Antenatal diagnosis and management of congenital cystic adenomatoid malformation. Semin Fetal Neonatal Med. 2007; 12(6):477-81. doi: 10.1016/j.siny.2007.06.009. PMID: 17950681.

12. Schrey S, Kelly EN, Langer JC, Davies GA, Windrim R, Seaward $P G$, et al. Fetal thoracoamniotic shunting for large macrocystic congenital cystic adenomatoid malformations of the lung. Ultrasound Obstet Gynecol. 2012; 39(5):515-20. doi: 10.1002/uog.11084. PMID: 22223532.

13. Smith RP, Illanes S, Denbow ML, Soothill PW. Outcome of fetal pleural effusions treated by thoracoamniotic shunting. Ultrasound Obstet Gynecol. 2005; 26(1):63-6. doi: 10.1002/uog.1883. PMID: 15861415.

14. Ong SS, Chan SY, Ewer AK, Jones M, Young P, Kilby MD. Laser ablation of foetal microcystic lung lesion: successful outcome and rationale for its use. Fetal Diagn Ther. 2006;21(5):471-4. doi: 10.1159/000093891. PMID: 16912498.

15. Min JY, Won HS, Lee MY, Suk HJ, Shim JY, Lee PR, Kim A. Intrauterine therapy for macrocystic congenital cystic adenomatoid malformation of the lung. Obstet Gynecol Sci. 2014; 57(2):102-8. doi: 10.5468/ogs.2014.57.2.102. PMID: 24678482; PMCID: PMC3965692.

16. Fan D, Wu S, Wang R, Huang Y, Fu Y, Ai W, et al. Successfully treated congenital cystic adenomatoid malformation by open fetal surgery: A care-compliant case report of a 5 -year follow-up and review of the literature. Medicine (Baltimore). 2017; 96(2):e5865.

doi: 10.1097/MD.0000000000005865. PMID: 28079822; PMCID: PMC5266184.

17. Tsao K, Hawgood S, Vu L, Hirose S, Sydorak R, Albanese CT, Farmer DL, et al. Resolution of hydrops fetalis in congenital cystic adenomatoid malformation after prenatal steroid therapy. J Pediatr Surg. 2003; 38(3):508-10. doi: 10.1053/jpsu.2003.50089. PMID: 12632377.

18. Peranteau WH, Wilson RD, Liechty KW, Johnson MP, Bebbington MW, Hedrick HL, et al. Effect of maternal betamethasone administration on prenatal congenital cystic adenomatoid malformation growth and fetal survival. Fetal Diagn Ther. 2007;22(5):365-71. doi: 10.1159/000103298. PMID: 17556826.

19. Thakkar HS, Durell J, Chakraborty S, Tingle BL, Choi A, Fowler DJ, et al. Antenatally Detected Congenital Pulmonary Airway Malformations: The Oxford Experience. Eur J Pediatr Surg. 2017; 27(4):324-329. doi: 10.1055/s0036-1593379. PMID: 27723920. 
20. Adams S, Jobson M, Sangnawakij P, Heetun A, Thaventhiran $A$, Johal $N$, et al. Does thoracoscopy have advantages over open surgery for asymptomatic congenital lung malformations? An analysis of 1626 resections. J Pediatr Surg. 2017; 52(2):247-251. doi: 10.1016/j. jpedsurg.2016.11.014. PMID: 27889066.

21. Kim HK, Choi YS, Kim K, Shim YM, Ku GW, Ahn KM, et al. Treatment of congenital cystic adenomatoid malformation: should lobectomy always be performed? Ann Thorac Surg. 2008; 86(1):249-53.

doi:10.1016/j. athoracsur.2008.01.036.PMID: 18573432.

22. Downard CD, Calkins CM, Williams RF, Renaud EJ, Jancelewicz T, Grabowski J, et al. Treatment of congenital pulmonary airway malformations: a systematic review from the APSA outcomes and evidence based practice committee. Pediatr Surg Int. 2017; 33(9):939-953. doi: 10.1007/s00383-017-4098-z. PMID: 28589256.

23. Eber E. Antenatal diagnosis of congenital thoracic malformations: early surgery, late surgery, or no surgery? Semin Respir Crit Care Med. 2007; 28(3):355-66. doi: 10.1055/s-2007-981656. PMID: 17562505.

24. Laje P, Liechty KW. Postnatal management and outcome of prenatally diagnosed lung lesions. Prenat Diagn. 2008; 28(7):612-8. doi: 10.1002/pd.1966. PMID: 18330859.

25. Naito $Y$, Beres A, Lapidus-Krol E, Ratjen F, Langer JC. Does earlier lobectomy result in better long-term pulmonary function in children with congenital lung anomalies? A prospective study. J Pediatr Surg. 2012; 47(5):852-6. doi: 10.1016/j.jpedsurg.2012.01.037. PMID: 22595560.

26. Keijzer R, Chiu PP, Ratjen F, Langer JC. Pulmonary function after early vs late lobectomy during childhood: a preliminary study. J Pediatr Surg. 2009; 44(5):893-5. doi: 10.1016/j.jpedsurg.2009.01.021. PMID: 19433164.

27. Di Prima FA, Bellia A, Inclimona G, Grasso F, Teresa M, Cassaro MN. Antenatally diagnosed congenital cystic adenomatoid malformations (CCAM): Research Review. J Prenat Med. 2012; 6(2):22-30. PMID: 22905308; PMCID: PMC3421952.

28. Kapralik J, Wayne C, Chan E, Nasr A. Surgical versus conservative management of congenital pulmonary airway malformation in children: A systematic review and meta-analysis. J Pediatr Surg. 2016; 51(3):508-12. doi: 10.1016/j.jpedsurg.2015.11.022. PMID: 26775193.
29. Cook J, Chitty LS, De Coppi P, Ashworth M, Wallis C. The natural history of prenatally diagnosed congenital cystic lung lesions: Iong-term follow-up of 119 cases. Arch Dis Child. 2017; 102(9):798-803.

doi:10.1136/archdischild-2016-311233.PMID: 28584070.

30. Rothenberg SS. Experience with thoracoscopic lobectomy in infants and children. J Pediatr Surg. 2003; 38(1):1024. doi: 10.1053/jpsu.2003.50020. PMID: 12592629.

31. Laberge JM, Puligandla P, Flageole H. Asymptomatic congenital lung malformations. Semin Pediatr Surg. 2005; 14(1):16-33.

doi:10.1053/j. sempedsurg.2004.10.022.PMID: 15770585.

32. Singh R, Davenport M. The argument for operative approach to asymptomatic lung lesions. Semin Pediatr Surg. 2015; 24(4):187-95. doi:10.1053/j. sempedsurg.2015.02.003.PMID: 26051052.

33. Feinberg A, Hall NJ, Williams GM, Schultz KA, Miniati D, Hill DA, et al. Can congenital pulmonary airway malformation be distinguished from Type I pleuropulmonary blastoma based on clinical and radiological features? J Pediatr Surg. 2016; 51(1):33-7. doi: 10.1016/j. jpedsurg.2015.10.019.PMID:26561249;PMCID: PMC5031236.

34. Sáez B J, Pattillo S JC, Vuletin S JF. Deformación de la pared torácica como presentación de un blastoma pleuropulmonar, caso clínico [Pleuro-pulmonary blastoma presenting as a chest wall deformity: a case report]. Rev Chil Pediatr. 2018; 89(2):231-235. Spanish.doi:10.4067/S0370-41062018000200231.PMID: 29799891.

35. Stanton M. The argument for a non-operative approach to asymptomatic lung lesions. Semin Pediatr Surg. 2015; 24(4):183-6. doi:10.1053/j.sempedsurg.2015.01.014.PMID: 26051051.

36. Wong KKY, Flake AW, Tibboel D, Rottier RJ, Tam PKH. Congenital pulmonary airway malformation: advances and controversies. Lancet Child Adolesc Health. 2018;2 (4):290-297. doi:10.1016/S2352-4642(18)30035-X. PMID: 30169300. 